\title{
Quantum Biology
}

\author{
$\operatorname{Jim}$ Al Khalili, ${ }^{a^{*}}$ Samuele Lilliu
}

\begin{abstract}
Prof. Jim-Al-Khalili explains the emerging field of quantum biology and discusses how this new discipline has developed from its inception in the 1920s until fruition in the late 1990s with the discovery of quantum effects in magnetoreception, olfaction, enzyme catalysis, and photosynthesis. See video at https://youtu.be/asps5mZ4Kp8
\end{abstract}

\section{Quantum Biology in a Nutshell}

If I was to define quantum biology, it is not what many people might think, that at the very deepest level, if you look into a living system, a living cell, down to the level of the molecules and atoms then you hit the quantum world, because that would be true for life as well as for inanimate matter, where the quantum rules kick in. Quantum biology, as we define it today, means exploring the mechanisms and phenomena that rely on non-trivial quantum effects within living cells. By non-trivial I mean quantum tunnelling, long lived quantum coherence and superposition, quantum entanglement. These are surprising effects that we are now seeing taking place within living organisms. That is quantum biology. ${ }^{1-3}$

\section{The Origins of Quantum Biology}

We tend to think about quantum biology as being quite a new area of interdisciplinary science and in many ways it is. But actually it has rather old origins, going all the way back to the early 1930s. In fact we can even trace it back to a particular lecture that Niels Bohr gave at a conference in $1929 .{ }^{4}$ He hinted at the idea, as many quantum pioneers were doing back then, that maybe quantum mechanics holds the key to so much of science and the fact that quantum mechanics, in their opinion, solved the problems of physics and chemistry, they arrogantly then assumed that it could also be used to tackle the mystery of life itself. Bohr was one of these early quantum pioneers, who suggested that maybe quantum mechanics could play a role. He inspired other physicists, particularly people like Max Delbrück, ${ }^{5}$ who then actually changed field and became a biophysicist working in molecular biology and also Pascual Jordan.

Jordan is most famous because he was one of the authors on the classic papers on quantum mechanics, on matrix mechanics, with Max Born and Werner Heisenberg in Göttingen. ${ }^{6-8}$ Jordan, I guess, is less known that Born and Heisenberg, but he is certainly one of the names of the quantum pioneers of the 1920s. Jordan, really, in a sense, was the founder of the field of quantum biology. He was looking for rules from the quantum world such as indeterminism, complementarity, ideas that he had developed by studying under Bohr, and whether they applied and played a vital role in life. In fact Pascual Jordan probably published the very first paper on quantum biology, back in 1932, advocating some of his ideas about how quantum mechanics and the act of observation and so on lead to the phenomena of life, which were still in some sense mysterious. ${ }^{9}$ The problem of course is Pascual Jordan's political views were rather unpleasant, he was a Nazi, and he wasn't just one of those people in 1930s Germany who kept their head down, who maybe didn't speak out against the government. No, he was a fully paid-up fascist. And so after the war with his reputation of course in ruin, quantum biology also in some sense was tainted by the shadow of being championed by Pascual Jordan. ${ }^{10}$

However there were others who still believed there was some-

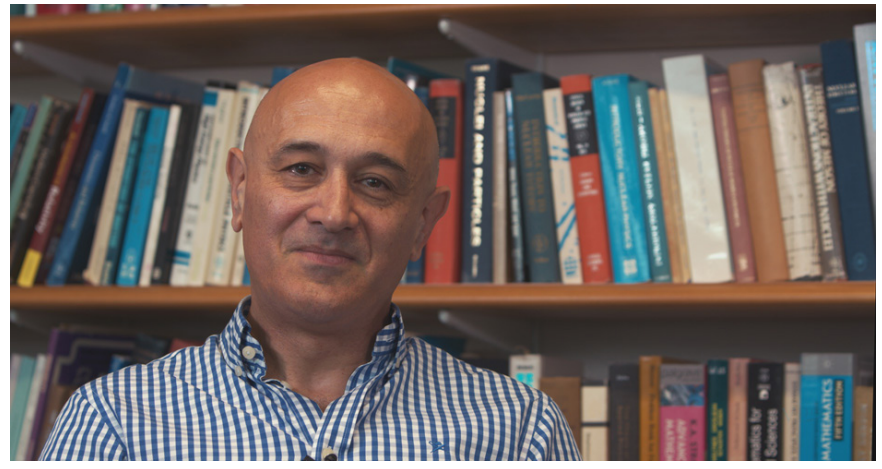

Figure 1 | Prof. Jim Al-Khalili, Distinguished Chair, Professor of Physics, Professor of Public Engagement in Science, Centre Director (University of Surrey)

thing in this idea that quantum mechanics could play a role in explaining life. So in the early 1930s the Cambridge Theoretical Biology Club was formed and it contained some of the greatest thinkers in Cambridge of that day. ${ }^{1}$ Even people life the great evolutionary biologist J.B.S. Haldane, ${ }^{11}$ many philosophers, many mathematicians, as well as physicists and biologists. The Cambridge Theoretical Biology Club essentially, I think, advocated a view, which we would call organicism.

Organicism, was half way between the other rather extreme schools of thought, which they believed were wrong when it came to describing life. ${ }^{12}$ On one hand you had the mechanistical, reductionist view of life, that living systems were highly ordered, maintain low entropy, but essentially we are steam engines. Give us energy we use that useful low entropy energy to maintain the order within the systems of life. That was the mechanistical view.

On the other extreme was vitalism. ${ }^{13}$ The vitalists had been discredited by the $20^{\text {th }}$ century really, because they believed there was some magical spark that endowed life with whatever makes life special, that differentiates between living and non-living matter.

So the organicists said "No, there is something more to life, than just sort of machines, you can't get to understand it with what we currently know, building on sort of Newtonian laws for example". But on the other hand there is, they argued, something special about life, maybe presumably some as yet undiscovered or not particularly well understood laws of physics and chemistry that are required to explain biology.

If I were pushed I'd say in some sense that is what most quantum biologists, certainly those physicists, chemists, biologists, spectroscopists, who work in quantum biology - there isn't really such a thing as quantum biology - this is what they would argue today, that there are laws of physics and chemistry as yet to be understood that would be needed to explain life.

a Department of Physics, University of Surrey, Guildford GU2 7XH, UK ( j.al-khalili@surrey.ac.uk), b Bullaki Itd, London, WC2H 9JQ, UK. 
Of course in addition to that group in Cambridge in 1930s there were others. Certainly for example, among the quantum pioneers, Erwin Schrödinger should really be mentioned because he published a very famous book called What is Life in 1944, in which he proposed that maybe the order of living systems is akin to inanimate matter at very low temperature. ${ }^{14}$ We know that when you drop down to near absolute zero quantum effects kick in, like superconductivity or super fluidity, when the thermodynamic random agitation of atoms and molecules can be calmed down and you allow for quantum effects to persist. We see that in inanimate matter at low temperature. Schrödinger was advocating that maybe living matter with its low entropy highly ordered states is akin to inanimate matter at low temperature therefore it also is the way it is because of quantum effects and quantum phenomena. He talked about aperiodic crystals, which of course is what DNA is essentially, it's an aperiodic crystal. So the basic fundamental building blocks of life do somehow have a requirement to be explained by laws of physics and in particular quantum physics.

\section{Quantum Biology after the Discovery of the DNA}

The one thing that we have to remember is that quantum mechanics and then developing in quantum field theory and so on was developing in parallel with the new areas of biology, genetics and molecular biology. The geneticists and molecular biologists by the 1930s and 1940s and indeed 1950s, when the double helix structure was discovered, really felt they had no need for quantum mechanics, they were so successful. They were learning so much about the molecular structure within living systems. They saw no requirement to bring in the strangeness of quantum mechanics. So to a large extent quantum biology really sort of went into the background. Particularly after the discovery of the double helix of DNA, spectroscopists and molecular biologists really were learning so much more about the building blocks of the cell, the instruction manual of life, they had no room for quantum superposition and the measurement problem, the uncertainty principle, and on all that silly business, they would leave that to physicists.

At the same time physicists had their hands full. We've also been very successful in the $20^{\text {th }}$ century from quantum mechanics to quantum field theory, nuclear and particle physics developed, we learned about the building blocks of matter. On the theoretical side we started looking at how we unify the different ideas, the different forces of nature. Quantum field theory itself then evolved into quantum electrodynamics, quantum chromodynamics by the 1960s and 1970s. We were building bigger and bigger accelerators to look at smaller and smaller constituents of matter.

Physicists didn't want to go and look at the messy world of biology. Biologists didn't have the quantum mechanical background to apply some of this hard maths to the processes of life. So until all the way for several decades, probably until the 1990s very little was done.

Quantum biology was seen as an outside, rather controversial, somehow wacky area of science. Particularly when you think about some of the ideas that grew up during the late 1960s, late 1970s, when people were using quantum mechanics to describe all sorts of strange phenomena such as telepathy or ideas in pseudo-science. Quantum mechanics developed this mystical arm.

One of the most famous examples was the work of Roger Penrose and Stuart Hameroff. They proposed a mechanism that they argued explained the nature of consciousness. ${ }^{15}$ The idea is that there were these proteins within the neurons of the brain that could exist in a quantum superposition of two configurations and when enough of them became entangled together that's when consciousness switched on. There was some brief excitement about this idea initially, but I think very quickly most scientists said: "No, hang on a minute, just because quantum mechanics is mysterious and we don't understand it and consciousness is mysterious and we don't under- stand it, it doesn't mean that the two have to be connected". And so that was another reason why people were rather nervous about approaching some of the ideas in quantum biology.

That changed. In the 1990s suddenly there were experimental techniques using fast pulsed lasers, 2D spectroscopy, where you could pump biomolecules, excite them and see how they decay. And suddenly some of these experiments were beginning to show that there were quantum effects going on, long living coherence, long lived interference effects that you couldn't explain otherwise. Think of the two slits experiment in quantum mechanics, firing a beam of particles, photons or electrons, through the two slits and you see the interference pattern. Even when you fire one at the time, you can't explain that interference pattern using classical mechanics, you need quantum mechanics. Well, they were seeing the equivalent of that taking place in certain special mechanisms with living cells, for example the way enzymes transfer particles from one part of the molecule to another, electrons and later even protons, 2000 times more massive than electrons, they were seeing these protons quantum tunnel from one place to another. ${ }^{16-18}$

\section{Quantum Biology in the $21^{\text {st }}$ Century}

I became interested in this field of quantum biology in the late nineties, but only as a hobby. I wasn't taking it seriously. My background is theoretical nuclear physics. So I spent my career modelling nuclear reactions, quantum scattering theories, where we can compare with experiments and we can develop and advance our theories.

But Johnjoe McFadden, who is a molecular biologist and a colleague of mine here at the University of Surrey, this was in 1997, he came to the physics department and he gave a seminar on an idea that he had. He admitted that it was probably a wacky idea. That there is a certain type of mutation called adaptive mutations, in which for example bacteria, E. Coli in this case that he was looking at, had the option of mutating in one direction or another and without any help from the surrounding environment that should be random, fifty-fifty. But if their surrounding environment contained, in this case, glucose sort of energy, one of those mutated states could take advantage of but the other couldn't, suddenly you saw more mutations going towards the side that could utilise the food in its environment. And this was a puzzle. How that can it know in advance, before it mutates it doesn't know there is glucose in the environment. "If I mutate in this way I can feed a multiply and make many copies of myself, and if I go in the other direction I can't utilise the glucose and I'll die".

The idea that Johnjoe McFadden had was that somehow some biomolecule within the E. Coli bacteria could exist in a superposition of two states or the genetic mutation could exist in a superposition of two states and it maintained that superposition until the time when it could, I guess, be measured by its environment, when it decoheres. It will happen in two different ways. ${ }^{19}$ It was a very handwavy idea and essentially most of the physics department here at Surrey who listened to his talk dismissed his idea out of hand, partly because it was crazy, and partly because he was a biologist trying to tell us physicists about quantum mechanics.

I was intrigued enough that I spoke to Johnjoe about this afterwards and thereafter began a collaboration that has now lasted over two decades between us, in which we looked at ideas of quantum mechanics in biology and as our interest has grown so has the field of quantum biology. ${ }^{20}$

Other examples have been discovered, still controversial, still open to be dismissed as wrong, but nevertheless examples published in top journals such as Science and Nature, ${ }^{21-23}$ which suggest that something quantum mechanical is going on inside living cells, whether it's in photosynthesis, in whether it's in enzyme catalysis, ${ }^{16-18}$ whether it's in mutations of DNA, ${ }^{19,24}$ even more controversially the way we smell, the theories of olfaction, ${ }^{25}$ or magnetoreception, the way certain animals can sense the Earth's magnetic field, the chem- 
ical compass that allows them to detect the orientation of the field relies on quantum effects, quantum entanglement. ${ }^{26-28}$

So these are controversial ideas, they are speculative but they are hugely exciting. And we have yet to know for sure whether or not this is going to lead to something. For me as a theoretical physicist, what's exciting is that it has allowed me to move into this new field, open quantum systems. The idea that when you are solving a problem a problem in quantum mechanics you no longer just solve the Schrödinger Equation because your quantum system of interest is surrounded by an environment that plays a very important role and that's why people are sceptical about quantum biology. They say, "How can these delicate, ephemeral, short-lived quantum effects have any functional role at all in biology given that they exist in an environment that is warm, complex, messy. Surely decoherence kicks in within femtoseconds, but to play a biologically significant role they have to last for pico- or nanoseconds or even longer. And yet, it seems, there are hints that life has evolved the ability to maintain these quantum effects for long biologically significant periods of time. The noise of the environment that measures the system, that causes it to decohere or today we talk about a quantum system becoming increasingly entangled with its surrounding environment, rather than killing off quantum effects it seems to be resonating with it, it seems maintaining it, there seems to be different kinds of noise, that we have to now consider. So from a theoretical point of view, this is a hugely exciting area, it borders on questions like what is the measurement problem, the questions about in foundations of quantum mechanics. ${ }^{29}$

\section{Potential Applications}

Studying these phenomena in quantum biology of course is more than just intellectual curiosity. If we think about some of the big areas that are funded in research today, particularly here in the UK, I can think of two, one is quantum technologies, the idea of utilising some of the non-trivial quantum mechanics to develop new instruments and new techniques and so on, new sensors. A lot of money is going to quantum technologies and has nothing to do with biology. On the other hand you have synthetic biology, developing machines that rely on the machinery of life. Quantum biology is somehow the bridge, I argue, between synthetic biology and quantum technologies. If some of these mechanisms that we are now seeing in living systems like long lived coherence in photosynthesis like quantum tunnelling in DNA, if they turn out to be true and, it is not magic, life has had nearly 4 billion years to perfect all its trickery, if utilising the rules of the quantum world gave life an advantage over classical rules it would have used them. So therefore, can we learn, if life has figured out some of these tricks, can we learn from life and develop our own ideas? Will this have a bearing on the work in developing quantum computers? Will it have a bearing on work developing new quantum magnetic sensors? So there are all sorts of technologies that might be advanced, maybe developing new types of photovoltaic cells, if plants and bacteria in their photosynthesis have used a very clever trick from the quantum world maybe we can copy that to help our advances in our technologies.

\section{Leverhulme Quantum Biology Doctoral Training Centre (QB-DTC)}

Now here in Surrey, Johnjoe and I have finally become very serious about quantum biology and we now have doctoral training centre, funded by the Leverhulme Trust, which is a charity, to take on PhD students. ${ }^{30}$ We now have an interdisciplinary group containing theoretical physicists, computational chemists, molecular biologists and geneticists, all working together, bringing together people from across the university in different fields, looking at different aspects of quantum biology. It may end up really not being anything but for me it's such an important question and the nature of life is still mysterious enough that I think it's too important not to look at seriously.

\section{References}

1 J. McFadden \& J. Al-Khalili. The origins of quantum biology. Proceedings of the Royal Society A: Mathematical, Physical and Engineering Sciences 474, 20180674, (2018).

2 G. R. Fleming, G. D. Scholes \& Y.-C. Cheng. Quantum effects in biology. Procedia Chemistry 3, 38-57, (2011).

3 G. D. Scholes, G. R. Fleming, L. X. Chen, A. Aspuru-Guzik, A. Buchleitner, D. F. Coker, G. S. Engel, R. van Grondelle, A. Ishizaki, D. M. Jonas, J. S. Lundeen, J. K. McCusker, S. Mukamel, J. P. Ogilvie, A. Olaya-Castro, M. A. Ratner, F. C. Spano, K. B. Whaley \& X. Zhu. Using coherence to enhance function in chemical and biophysical systems. Nature 543, 647-656, (2017).

4 K. J. Foundations of Quantum Physics II (1933-1958), https://books.google. co.uk/books?hl=en\&lr=\&id=yet5P7f 630 C\&oi=fnd\&pg $=$ PP $1 \&$ ots $=$ gCmsGlIwb3\&sig=iLMnNaTzG5QDbNt3CXiFNe0d6f8\&redir esc $=\mathrm{y} \# \mathrm{v}=$ onepage\&q\&f=false, (2013).

5 T.-R. NW, Z. KG \& D. M. On the Nature of gene mutation and gene structure, https://www.ini.uzh.ch/ tobi/fun/max/timofeeffZimmerDelbruck1935.pdf, (1935).

6 M. Born \& P. Jordan. Zur Quantentheorie aperiodischer Vorgänge. Zeitschrift für Physik 33, 479-505, (1925).

7 M. Born \& P. Jordan. Zur Quantenmechanik. Zeitschrift für Physik 34, 858888, (1925).

8 M. Born, W. Heisenberg \& P. Jordan. Zur Quantenmechanik. II. Zeitschrift für Physik 35, 557-615, (1926).

9 P. Jordan. Die Quantenmechanik und die Grundprobleme der Biologie und Psychologie. Naturwissenschaften 20, 815-821, (1932).

10 P. Jordan. Die Physik und das Geheimnis des organischen Lebens, https://www. amazon.de/Die-Physik-Geheimnis-Organischen-Lebens/dp/B002/KIH3K, (1948).

11 J. B. S. Haldane. Quantum Mechanics as a Basis for Philosophy. Philosophy of Science 1, 78-98, (1934).

12 L. Bertalanffy. Kritische Theorie der Formbildung. (1931).

13 H. Bergson. Creative evolution, https://books.google.co.uk/books?hl=en\&l-

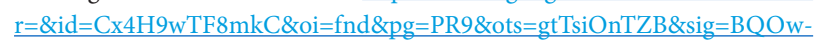

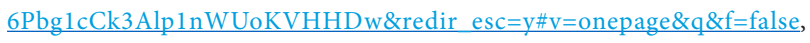
(1984).

14 E. Schrödinger. What is life? and other scientific essays, https://www.amazon. $\underline{\mathrm{com} / \text { What-life-Other-Scientific-Essays/dp/B000KU7940/ref=cm cr arp d }}$ product top?ie=UTF8, $(1956)$

15 S. Hameroff \& R. Penrose. Orchestrated reduction of quantum coherence in brain microtubules: A model for consciousness. Mathematics and Computers in Simulation 40, 453-480, (1996).

16 A. Kohen, R. Cannio, S. Bartolucci, J. P. Klinman \& J. P. Klinman. Enzyme dynamics and hydrogen tunnelling in a thermophilic alcohol dehydrogenase. Nature 399, 496-499, (1999).

17 C. C. Page, C. C. Moser, X. Chen \& P. L. Dutton. Natural engineering principles of electron tunnelling in biological oxidation-reduction. Nature 402, 47-52, (1999).

18 L. Masgrau, A. Roujeinikova, L. O. Johannissen, P. Hothi, J. Basran, K. E. Ranaghan, A. J. Mulholland, M. J. Sutcliffe, N. S. Scrutton \& D. Leys. Atomic Description of an Enzyme Reaction Dominated by Proton Tunneling. Science 312, 237, (2006).

19 J. McFadden \& J. Al-Khalili. A quantum mechanical model of adaptive mutation. Biosystems 50, 203-211, (1999).

20 J. McFadden \& J. Al-Khalili. Life on the edge: the coming of age of quantum biology, https://www.amazon.co.uk/Life-Edge-Coming-Quantum-Biology/ dp $/ 0552778079 /$ ref $=$ sr_1_1?dchild $=1$ \&keywords=9780552778077\&linkCode $=$ qs\& $q$ id $=1588875625 \&$ s $=$ books\&sr $=1-1$, (2016).

21 G. S. Engel, T. R. Calhoun, E. L. Read, T.-K. Ahn, T. Mančal, Y.-C. Cheng, R. E. Blankenship \& G. R. Fleming. Evidence for wavelike energy transfer through quantum coherence in photosynthetic systems. Nature 446, 782-786, (2007).

22 H. Lee, Y.-C. Cheng \& G. R. Fleming. Coherence Dynamics in Photosynthesis: Protein Protection of Excitonic Coherence. Science 316, 1462, (2007).

23 E. Collini, C. Y. Wong, K. E. Wilk, P. M. G. Curmi, P. Brumer \& G. D. Scholes. Coherently wired light-harvesting in photosynthetic marine algae at ambient temperature. Nature 463, 644-647, (2010). 
24 A. D. Godbeer, J. S. Al-Khalili \& P. D. Stevenson. Modelling proton tunnelling in the adenine-thymine base pair. Physical Chemistry Chemical Physics 17, 13034-13044, (2015)

25 L. Turin. A Spectroscopic Mechanism for Primary Olfactory Reception. Chemical Senses 21, 773-791, (1996).

26 J. Cai, G. G. Guerreschi \& H. J. Briegel. Quantum Control and Entanglement in a Chemical Compass. Physical Review Letters 104, 220502, (2010).

27 E. M. Gauger, E. Rieper, J. J. L. Morton, S. C. Benjamin \& V. Vedral. Sustained Quantum Coherence and Entanglement in the Avian Compass. Physical Review Letters 106, 040503, (2011).

28 T. Ritz, P. Thalau, J. B. Phillips, R. Wiltschko \& W. Wiltschko. Resonance effects indicate a radical-pair mechanism for avian magnetic compass. Nature 429, 177-180, (2004).

29 J. Cao, R. J. Cogdell, D. F. Coker, H.-G. Duan, J. Hauer, U. Kleinekathöfer, T. L. C. Jansen, T. Mančal, R. J. D. Miller, J. P. Ogilvie, V. I. Prokhorenko, T. Renger, H.-S. Tan, R. Tempelaar, M. Thorwart, E. Thyrhaug, S. Westenhoff \& D. Zig mantas. Quantum biology revisited. Science Advances 6, eaaz4888, (2020).

30 L. Q. B. D. T. C. (QB-DTC). Leverhulme Quantum Biology Doctoral Training Centre (QB-DTC) https://www.surrey.ac.uk/leverhulme-quantum-biology-doctoral-training-centre/about-us, (2020).

\section{Conflicts of Interest}

There are no conflicts to declare.

\section{Acknowledgements}

SL wishes to thank Dalitso Njolinjo for logistics support.

\section{Author Contributions}

JK was interviewed by SL, who wrote this manuscript.

\section{Keywords}

Quantum biology, Quantum tunnelling, Superposition, Quantum Entanglement, Biology (Field Of Study), Two-slit experiment, Magnetic field, Photosynthesis, Physics, Pascual Jordan, Erwin Schrödinger, Consciousness, magnetoreception, olfaction, enzyme catalysis.

\section{Article Information}

This article is licensed by Jim Al-Khalili et al. under a Creative Commons Attribution 4.0 International License (CC BY 4.0). 